\title{
A WSN positioning algorithm based on 3D discrete chaotic mapping
}

\author{
Tu Li ${ }^{1}$, Wang Yan ${ }^{2 *}$, Li Ping ${ }^{1}$ and Peng Fang ${ }^{1}$
}

\begin{abstract}
Wireless sensor networks are featured by restricted network resources, which is quite possible to result in low positioning precision and serious time delay in positioning, accordingly, the overall network positioning quality may be reduced; to improve the positioning precision of WSN, based on the DV-HOP positioning algorithm, two aspects of the node positioning were improved from the error precision and least square estimation; thus, a WSN positioning algorithm based on 3D discrete chaotic mapping was proposed: first, a 3D discrete chaotic mapping was constructed, the Chaos Optimization Algorithm was introduced into the positioning error precision calculation, and the unknown nodes were positioned by introducing the least square estimation; second, a simulation experiment of new algorithm was performed from the aspects of communication radius and topological structure. The experimental results showed that the algorithm proposed in this paper can effectively reduce the positioning error caused by calculation and improved the positioning precision. Further, based on the algorithm of this paper, the moving mechanism could be introduced to make dynamic planning for overall network resources, so that the energy cost of the algorithm of this paper in the confirmation process of WSN network terminal could be further reduced to make the algorithm more valuable in engineering field.
\end{abstract}

Keywords: Wireless sensor networks, Node positioning, Chaos optimization, Least squares estimation

\section{Introduction}

With the development of electronic technology and Internet communication technology, the cost of collecting and processing multimedia information (such as images and audio) is getting lower and lower, and the scalar information collected by traditional sensor networks (such as temperature, humidity, pressure) becomes unable to satisfy the diversified application of the information, therefore, the wireless sensor networks (WSN) emerged [1-7]. Featured by low-cost, rapid network establishment, dynamic topology, and multi-hop routing, WSN is widely applied in industries such as environmental monitoring and industrial technology. WSN integrates the sensor technology, embedded computing technology, modern network technology, and wireless communication technique and is able to perform the real-time monitoring and collection of monitoring objects information through various microsensors. The information is sent in a wireless way and transmitted to user terminal through ad hoc and multi-hop network, so as to achieve the targeted information acquisition $[8,9]$. A typical network topology is shown in Fig. 1.

WSN is a multi-hop network composed of micro multimedia sensor nodes which are interlinked and battery powered. From Fig. 1, it can be found that each sensor node is able to process the multimedia data for being equipped with wireless transceiver module, and the volume, power dissipation, operation and storage capability, and wireless communication distance of the nodes are all available to be customized based on application needs. The network nodes in WSN are randomly distributed, with the control center node, without fixed organization structure; ad hoc networks are conducted by information interaction and intercoordination of nodes in networks [9, 10]. The sensor nodes are set in site to observe the physical phenomenon; in most cases, only the data included position information is of practical significance; therefore, the node positioning technology plays a very important role in WSN system.

\footnotetext{
* Correspondence: 84993894@qq.com

${ }^{2}$ School of Information and Electronic Engineering, Hunan City University,

Yiyang, China

Full list of author information is available at the end of the article
} 


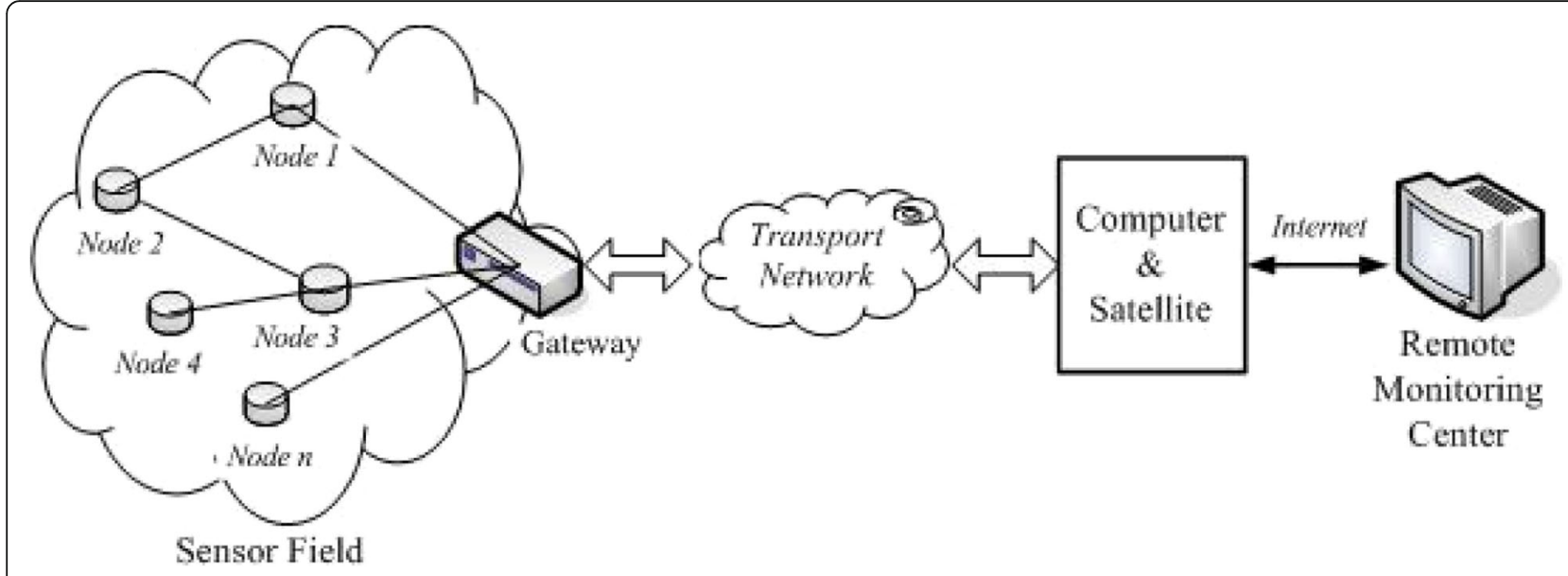

Fig. 1 Topology of wireless sensor networks

\section{WSN node positioning methods}

The node positioning system includes distance measurement, position calculation, and positioning algorithm which are explained below respectively:

1. Distance (angle) measurement: information on the distance (angle) between nodes is acquired by physical distance measurement or multi-hop links;

2. Position estimation: the unknown nodes' position can be estimated through the information on the distance (angle). If there are anchor nodes, the unknown nodes' position can be estimated through calculation;

3. Positioning algorithm: selecting right positioning algorithm according to the WSN application site. The position of all unknown nodes within the monitored area can be acquired through positioning algorithm on the basis of previous information.

\subsection{Computing methods of WSN node positioning}

The computing methods of WSN node positioning are divided three classes: trilateration [11], angular measurement [12], and maximum likelihood method [13, 14]. The maximum likelihood method is also named multilateral measurement, when the data satisfy the Gaussian distribution, it is equal to the least square method. To solve the overdetermined equation, the maximum likelihood method needs the known anchor nodes of $n$ coordinates position to calculate the coordinate position of the positioned node. The principles of maximum likelihood method are presented below:

Assuming that the measured distance between the positioned node $p(x, y)$ and the anchor nodes within the measuring radius $p_{1}\left(x_{1}, y_{1}\right), p_{2}\left(x_{2}, y_{2}\right), \ldots, p_{n}\left(x_{n}, y_{n}\right)$ are $d_{1}, d_{2}, d_{3} \ldots$, respectively, the following Eq. (1) can be obtained from the distance formula between two points:

$$
\left\{\begin{array}{c}
\left(x-x_{1}\right)^{2}+\left(y-y_{1}\right)^{2}=d_{1}^{2} \\
\left(x-x_{2}\right)^{2}+\left(y-y_{2}\right)^{2}=d_{2}^{2} \\
\cdots \\
\left(x-x_{n}\right)^{2}+\left(y-y_{n}\right)^{2}=d_{n}^{2}
\end{array}\right.
$$

Equation (2) can be obtained by subtracting the n-1th equation from $n$th equation in proper order:

$$
\begin{aligned}
& {\left[\begin{array}{l}
x_{n} \\
y_{n}
\end{array}\right] x\left[\begin{array}{cc}
2\left(x_{1}-x_{n}\right) & 2\left(y_{1}-y_{n}\right) \\
\ldots & \\
2\left(x_{n-1}-x_{n}\right) & 2\left(y_{n-1}-y_{n}\right)
\end{array}\right] A} \\
& =\left[\begin{array}{c}
{\left[x_{1}^{2}-x_{n}^{2}+y_{1}^{2}-y_{n}^{2}+d_{n}^{2}-d_{1}^{2}\right]} \\
\ldots \\
x_{n-1}^{2}-x_{n}^{2}+y_{n-1}^{2}-y_{n}^{2}+d_{n}^{2}-d_{n-1}^{2}
\end{array}\right] b
\end{aligned}
$$

The solution vector of position of node $\mathrm{P} X=\left(A^{T} A\right)^{-1} A^{T} b$ can be obtained by using the least mean square error. Compared with the trilateration, the maximum likelihood method has higher positioning precision, and with superfluous term, the fault freedom is excellent. The maximum likehood is shown in Fig. 2.

\subsection{WSN node positioning algorithms}

The WSN node positioning algorithms are divided in the following classes:

1. Positioning algorithms based on and regardless of distance measurement

The range-based positioning algorithm calculates the node position by measuring the distance between nodes or the angle information and mainly includes the received signal strength indicator-based algorithm (RSSI) [15], time of advent-based positioning algorithm (TOA) [16], time difference of advent-based positioning 


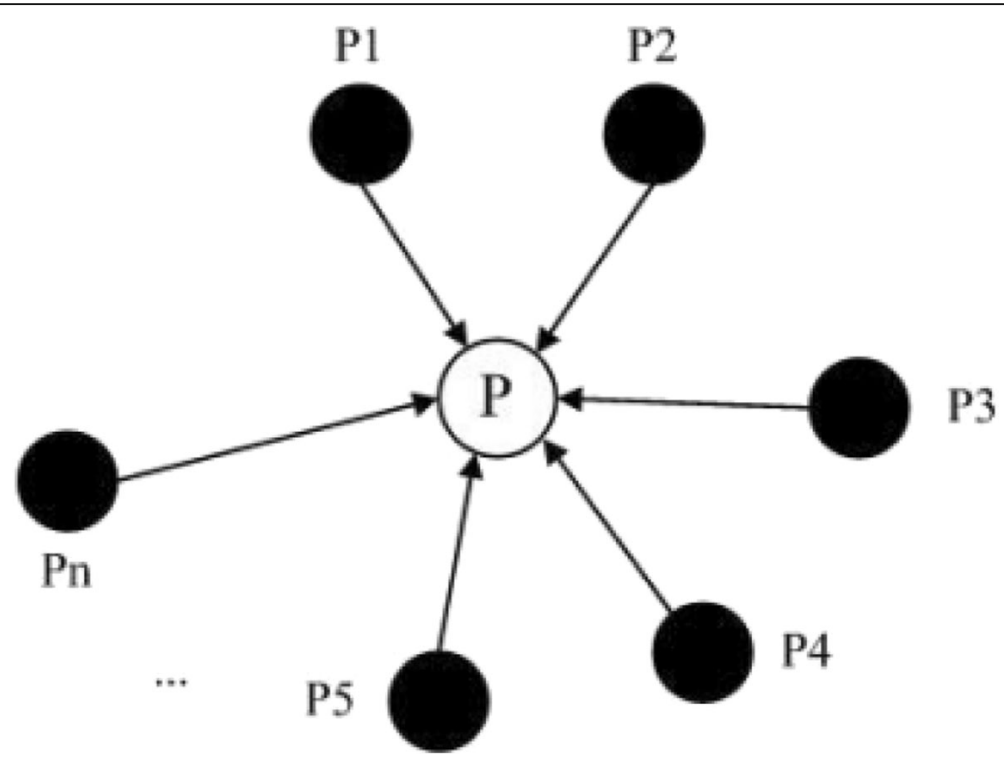

Fig. 2 The maximum likelihood method

algorithm (TDOA) [17], and angle of advent-based positioning algorithm (AOA) [18]. These algorithms are featured by higher precision, higher requirements on hardware, higher cost, and being limited by distance measurement technology.

The positioning algorithms regardless of distance measurement acquire the distance between nodes through the fixed multi-hop communication relationship of WSN [19], so as to calculate the position of positioned node. They are featured by low cost and easy realization and are suitable for site with dense nodes.

2. Incremental positioning algorithms and concurrent positioning algorithms

The incremental positioning algorithms take the anchor node as core point, starting from the neighbor nodes of anchor node, to expand outwards. The node positioning are conducted one-by-one by choosing the correct computing method according to the distance between the positioned node and known node or the angle information; in current positioning algorithms, all the unknown nodes confirm their position based on the coordinate information of anchor node's position without order. In incremental positioning algorithms, the errors accumulate gradually, which results in lower and lower positioning precision; therefore, it is suitable for the sensor network where there are many nodes and little anchor nodes and with wide coverage area.

3. Positioning algorithms based on anchor node and positioning algorithms without anchor node
While using the positioning algorithms based on anchor node, the anchor node is taken as a reference, through corresponding positioning calculation, and the unknown nodes are able to acquire their position coordinate; while using the positioning algorithms without anchor node, the anchor node is not involved in positioning, but the relative coordinates of nodes are necessary to be confirmed. Next, the relative coordinates are combined to establish the system and the unknown nodes will have an overall relative coordinates after the positioning process ended.

\subsection{DV-HOP algorithm}

Distance vector (DV)-HOP algorithm means to express the distance between the anchor node and unknown nodes with the product of hop count and the average distance between hops [20]. The detailed steps are described below:

1. Storing the average hop count from anchor node acquired by all unknown nodes;

2. Calculating the average value of hop distance of each anchor node and transmitting to network;

$$
H_{i j}=\frac{\sum_{i \neq j} \sqrt{\left(x_{i}-x_{j}\right)^{2}+\left(y_{i}-y_{j}\right)^{2}}}{\sum_{i \neq j} c_{i j}}
$$

In Eq. (3), $\left(x_{i}, y_{i}\right)$ and $\left(x_{j}, y_{j}\right)$ represent the coordinates of anchor nodes $i$ and $j$, respectively, and $c_{i j}$ is the hop count between anchor nodes $i$ and $j$. 
3. According to the principle that three points determine a plane, the unknown nodes need the hop distance of at least $\mathrm{n}(n>3)$ anchor nodes to acquire the coordinates of their position.

$$
\left\{\begin{array}{c}
\sqrt{\left(x_{1}-x\right)^{2}+\left(y_{1}-y\right)^{2}}=l_{1} \\
\ldots \\
\sqrt{\left(x_{n}-x\right)^{2}+\left(y_{n}-y\right)^{2}}=l_{n}
\end{array}\right.
$$

There are two problems existing in the traditional DV-HOP algorithm, one is the error which occurred in the calculation of the average hop distance, and the other is the larger error of average hop distance while replacing the unknown nodes with anchor nodes.

\section{Improved DV-HOP algorithm}

In this paper, the average hop distance of the whole network in literature [19] is firstly used to replace the original average hop distance, where $n$ is the number of anchor nodes in the network, as shown in Eq. (5):

$$
F_{i}=\frac{\sum H_{i j}}{n}
$$

The error in hop distance of the whole network is acquired by using Eq. (5) to calculate the average hop distance of the whole network, as shown in Eq. (6):

$$
f=\frac{\sum_{i \neq j}\left|\sqrt{\left(x_{i}-x_{j}\right)^{2}+\left(y_{i}-y_{j}\right)^{2}}-F_{i} * c_{i j}\right|}{\min \left(\sum_{i \neq j} c_{i j}\right)}
$$

where the numerator represents the difference of the actual distance and estimated distance between any two anchor nodes in the whole network and the denominator represents the minimum hop count between any two anchor nodes.

\subsection{Chaotic system and its optimization algorithm}

The Chaos optimization algorithm is a new optimization technology and is advanced in numerical optimization. In this paper, after the distance between the anchor nodes is estimated, it is mapped to the variable space through chaos, so as to search the optimal solution by using the ergodicity and randomness of chaos variables.

Feigenbaum equation is a one-dimensional chaotic equation [21] and is defined below:

$$
x_{n+1}=\mu \sin \left(\pi x_{n}\right)
$$

where $-4 \leq x_{n} \leq 4, \mu \in(0,4)$, let the initial value $x_{1}$ be 0.5 ; Fig. 3 is acquired by iterating different parameters $\mu$ for 200 times.

When $\mu<0.315$, the iterative value $x_{n}$ converges to 0 ;

When $0.315<\mu<0.719$, the iterative value converges to a non-zero value, and the steady-state solution of system is the fixed point;

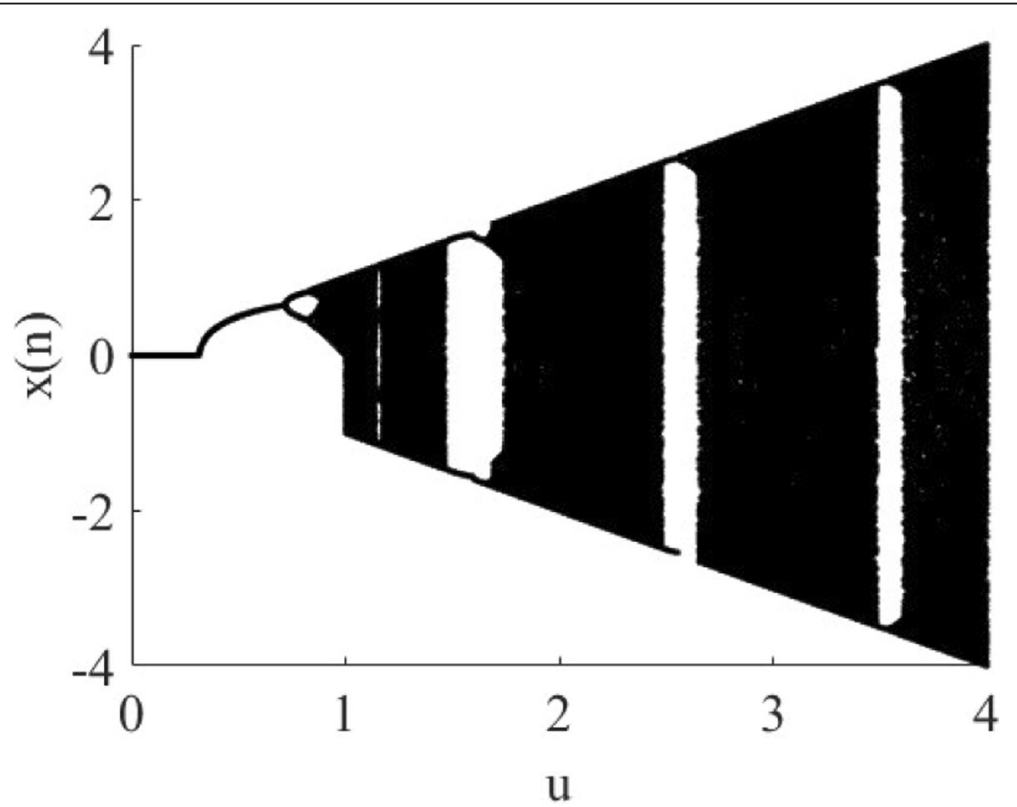

Fig. 3 Bifurcation of Feigenbaum mapping. The initial value $x_{1}$ is 0.5 ; the figure is acquired by iterating different parameters $\mu$ for 200 times 
When $\mu \geq 0.719$, the system becomes period doubling bifurcation, which means that two periods become four periods, and four periods become eight periods.

When $\mu \geq 0.870$, the iterative value is in a state of pseudo-random distribution, and the system becomes chaotic.

After the system became chaotic, there would be a blank area [22]; it could be found from Fig. 3 that in certain intervals of $\mu \in(1.4-1.8)$, the iterative value converges to certain fixed value and presents an obvious blank area. As shown in Fig. 4, when $\mu=1.63$, the system converges to two fixed non-zero value and becomes a periodic function without any chaotic features.

The blank area mapped by chaos may exist in all positions within the taking value interval of parameter $\mu$. When using the typical Feigenbaum equation in the WSN positioning, different parameters $\mu$ and initial value $x$ may be corresponding with the pathway of blank area, and because of the lack of sample space, the randomness of sequence may be quite bad and the ergodicity of system will be seriously influenced [22, 23].

Because the chaotic features of logistic chaos mapping are quite sensitive to initial value [24], the dynamic initial value can be used to make the iteration of chaos mapping performed in different pathways to avoid the blank area. In this paper, by using the Feigenbaum equation featured by secondary coupling, the three-dimensional Feigenbaum logistic mapping based on initial value disturbance is proposed. The equation changes the initial value $\omega_{n}$ through logistic mapping makes the iterative value in different chaotic pathways, so as to avoid the blank area.

The mapping of improved chaotic equation is shown in Eq. (8):

$$
\left\{\begin{array}{cc}
x_{n+1}=r_{1} \sin \left(6 w_{n} x_{n}\right)+u_{1} y_{n} z_{n} & r_{1}, u_{1} \in(0,1) \\
y_{n+1}=r_{2} \sin \left(6 w_{n} y_{n}\right)+u_{2} x_{n} z_{n} & r_{2}, u_{2} \in(0,1) \\
z_{n+1}=r_{3} \sin \left(6 w_{n} z_{n}\right)+u_{3} x_{n} y_{n} & r_{3}, u_{3} \in(0,1) \\
w_{n+1}=k w_{n}\left(1-w_{n}\right) & w_{n} \in(0,1)
\end{array}\right.
$$

Let the initial value $\mu_{1}=0.2 ; \mu_{2}=0.3 ; \mu_{3}=0.5 ; k=$ 3.93, $\omega 1=0.6 ; r_{1}=r_{2}=r_{3} \in(0,4)$, the iteration number is 300 . The results of the previous 100 iterations are abandoned, the iteration diagram which includes the control parameter $r$ and the iterative value of sequence $x$ of improved Feigenbaum mapping (the iterative diagram of sequence $y, z$ is similar to that in Fig. 5), as shown in Fig. 5:

When $r<0.315$, after multiple convergences, the iterative sequence value is equal to 0 ;

When $r<0.719$, the iterative sequence is a non-zero fixed value;

When $r>0.753$, the chaotic mapping has negative value and becomes chaotic, and the whole chaotic space has no blank area.

Choosing proper initial value for iterative computations, a sequence within the interval $[-1,1]$ is acquired by dividing the sequence $x$ by $r$ value. The results of

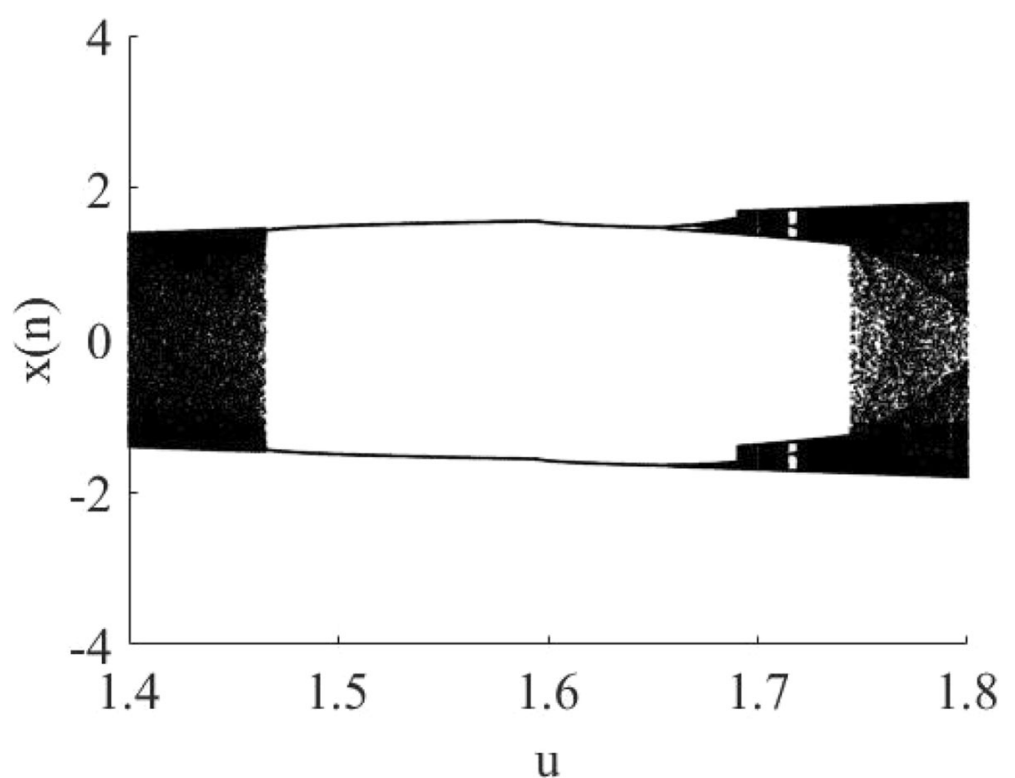

Fig. 4 The blank area of bifurcation of Feigenbaum mapping. The initial value $x_{1}$ is 0.5 , and parameter $\mu \in(1.4-1.8)$, Figure is acquired by iterating different parameters $\mu$ for 200 times, and it shows the blank area of bifurcation of Feigenbaum mapping 


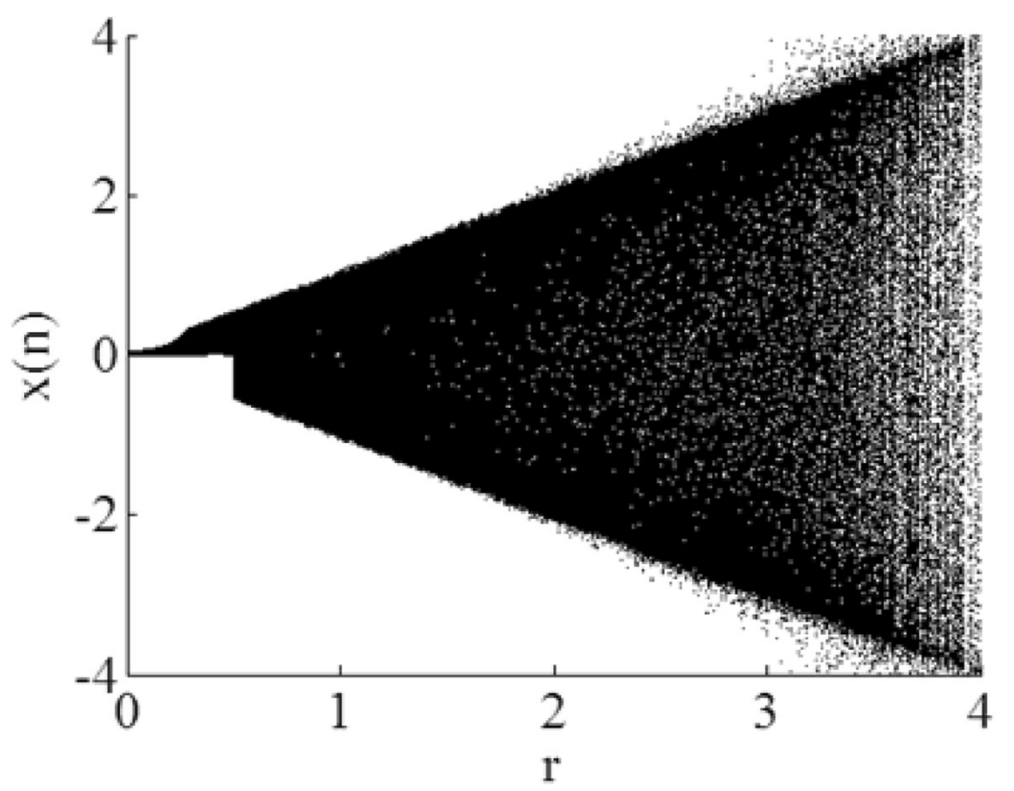

Fig. 5 Bifurcation of sequence $x$ of improved three-dimensional Feigenbaum equation. The initial value $x_{1}$ is 0.5 , and the parameters are $\mu_{1}=0.2$; $\mu_{2}=0.3 ; \mu_{3}=0.5 ; k=3.93, \omega_{1}=0.6 ; r_{1}=r_{2}=r_{3} \in(0,4)$, Figure is acquired by iterating different parameters $\mu$ for 200 times

Eq. (6) are mapped to the interval $[-1,1]$ by using the features of the logistic mapping function.

$$
L_{i j}=\frac{2\left(F_{\text {average }} c_{i j}-d_{i j \min }\right)}{d_{i j \max }+d_{i j \min }}
$$

According to the three-dimensional chaotic Eq. (8) and formula (9), new chaotic entity is acquired by adding the chaotic variables into searched entities. The newly acquired entity is transformed according to formula (10). Where $\mathrm{d} i \min$ and $\mathrm{d} i \max$ represent the minimum and maximum of range of anchor node, respectively.

$$
F_{i j}^{\prime}=\left(d_{i j \max }-d_{i j \min }\right) L_{i j}+\frac{1}{2}\left(d_{i j \max }-d_{i j \min }\right)
$$

Assuming that $X=\left(x_{1}, x_{2} \ldots x_{\mathrm{D}}\right)$ represents the set of the unknown nodes, $\left[a_{\mathrm{j}}, b_{\mathrm{j}}\right]$ represents the minimum and maximum of position range of unknown nodes, and it satisfies $x_{\mathrm{j}} \in\left[a_{\mathrm{j}}, b_{\mathrm{j}}\right]$, the reversed solution of $X$ is given by: $X^{*}=\left(x_{1}^{*}, x^{*}{ }_{2} \ldots x_{\mathrm{D}}^{*}\right)$. The unknown reverse value of nodes is calculated to better shrink the positioning information of space.

$$
\left\{\begin{array}{l}
X_{i j}^{*}=k\left(a_{j}(i)+b_{j}(i)\right)-X_{i j} \\
X_{i j}^{*}=\operatorname{random}\left(a_{j}(i), b_{j}(i)\right) \quad X_{i j} \notin\left[X_{\min }, X_{i \max }\right]
\end{array}\right.
$$

Formula (12) is acquired by solving reversely the formula (11):

$$
\begin{aligned}
& X_{i j}^{\prime \prime}=t\left(X_{\text {best }}+X_{\text {worst }}\right)-X_{i j}^{\prime} \\
& X_{i j}=\min \left(X_{i j}^{*}, X_{i j}^{\prime \prime}\right)
\end{aligned}
$$

Comparing the node position in formula (13), the minimum is selected as the reliable position of unknown nodes. $X_{i j}$ is further positioned by using the weighted least square estimation, and the formula (14) is acquired:

$$
X_{w l s}=\left(A^{T} W\right)^{-1} W B
$$

where $A$ is the weighting matrix and used to ensure that $X_{w l s}$ is agonic and $W$ is the symmetric matrix.

\subsection{Description of algorithm improvement}

The algorithm improvement includes four steps which are described below:

Step 1: Collecting the average hop count and position information of anchor nodes

Step 2: The anchor node acquires the average hop distance of other anchor nodes according to formulas 3 and 5, calculates the stability of the anchor node and surrounding anchor nodes, and informs the other nodes in the wireless sensor network

Step 3: The unknown nodes calculate the initial position with formula 6 based on the average hop distance of anchor nodes in Step 2

Step 4: The precise node position is calculated by substituting the unknown node position in Step 3 into the formulas 13 and 14 


\section{Simulation experiment}

Choosing proper initial value $u_{1}=0.2 ; u_{2}=0.3 ; u_{3}=0.5$; $r=3 ; k=3.93, w_{1}=0.6$, conducting iterative computations on chaotic equation, and adopting Matlab simulation experiment environment, the Colliner precision positioning (CPP) algorithm [25], which is widely applied in the control group, and the analysis are performed from four indexes of terminal position precision, positioning error precision, positioning timedelay, and energy consumption bandwidth. The specific simulation parameters are shown in Table 1:

\subsection{Terminal position precision}

Figure 6 showed the comparison of the algorithms proposed in this paper and by literature [25] and [27]. It can be known from the figure that with the continuous increase of network nodes, there is a little fluctuation on the terminal position precision of the algorithm in this paper, and precision is always higher, while a sharp fall appeared on that of the algorithms of literature [25] and [27]. The algorithm in this paper has higher precision because it introduced the self-adapting radio frequency inference which is able to ensure the channel holding at a higher level when there are fluctuations on network topology; while both the algorithms of literature [25] and [27] use the fixed channel distribution mode, once there are fluctuations on the network topology, the channel holding will be unavailable and the terminal position precision will decrease.

\subsection{Positioning error precision}

Setting an error formula, where $\left(x_{i}^{\prime}, y_{i}^{\prime}\right)$ and $\left(x^{\prime}, y^{\prime}\right)$ represent the estimated unknown nodes and the actual unknown nodes, respectively, $R$ is the communication radius of nodes, the results are shown in following formula [26]:

Table 1 Simulation parameters

\begin{tabular}{ll}
\hline Parameters & Numerical value \\
\hline Area of region & $100 \mathrm{~m} \times 100 \mathrm{~m}$ \\
Simulation period & 3600 \\
Subdomain amount & Less than 1024 \\
Nodes distribution method & Random distribution \\
Distribution density of nodes in subdomain & No higher than 30 \\
Communication radius of nodes signal & $20 \mathrm{~m}$ \\
Nodes cache capacity & $512 \mathrm{~KB}$ \\
Emission period of nodes radio-frequency signal & $60 \mathrm{~S}$ \\
Primary energy of nodes & $5-20 \mathrm{~J}$ \\
\hline
\end{tabular}

$$
e_{i}=\frac{\sum_{i \neq j} \sqrt{\left(x_{i}^{\prime}-x_{i}\right)^{2}+\left(y_{i}^{\prime}-y_{i}\right)^{2}}}{N \times R}
$$

In the experiment, the algorithms proposed in this paper and by literature [25] and [27] are compared for positioning error by setting different proportion of anchor nodes and communication radius. Each algorithm is operated for 100 times, and the results of three algorithms are compared based on average value.

Figure 7 showed the comparison of the positioning error precision of three algorithms with different nodes density; it can be known from the figure that with the continuous increase of network density, the positioning error precision of the algorithm in this paper is always higher than that of the control group, and this is because the algorithm in this paper uses the resource scheduling mode based on radio frequency inference and the WSN's demand of serving for resources is satisfied by fixing the service time when the node density increase continuously. While the algorithms proposed by literature [25] and [27] do not take the influence of density on network congestion into account, it is difficult to improve the quality assurance demand of WSN flow of service when there is a network congestion; therefore, the positioning error precision of literature [25] and [27] is much lower than that of this paper.

With two different communication radiuses, the node positioning error of the algorithm in this paper is fewer than that of two other algorithms. This indicates that the positioning precision of the algorithm in this paper is higher than two other algorithms. It can be found from the figure that the error is bigger when the proportion of anchor nodes is lower. The main reason is that the number of unknown nodes is much more than the anchor nodes, and the computational error occurs because the distance of many unknown nodes depends on few anchor nodes. In addition, the distance between the unknown nodes and the anchor nodes is far; there are many hops, which results in some influences on the average hop distance. And it can be found from Figs. 8 and 9 that with the increases of communication radius, the positioning error reduces, because the chaotic optimization which optimized the influence of communication radius on average hop distance to a certain extent is used in positioning error.

\subsection{Positioning time-delay}

Figure 10 showed the comparison of the positioning time-delay of the algorithms proposed in this paper and by literature [25] and [27]; it can be known from the figure that with the continuous increase of nodes density, the network is at high density node and low density node and the positioning time-delay index of 


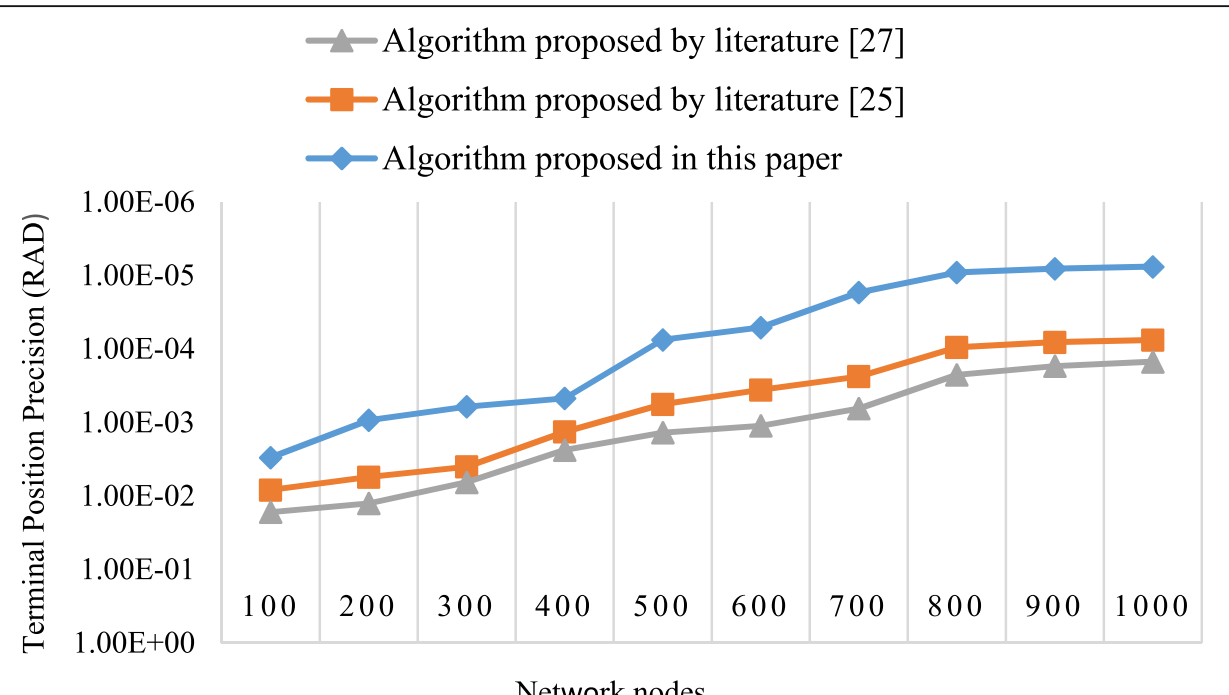

Network nodes

Fig. 6 Relationship between the terminal position precision and network nodes. Figure shows the comparison of the algorithms proposed in this paper and by literature [25] and [27]

the algorithm in this paper is always lower than that of the control group. The algorithm in this paper adjusts the service quality dynamically according to the current network situation; therefore, it has a lower positioning time-delay index and is not proportional to the changes of nodes density distribution, while the algorithms of literature [25] and [27] conduct the resource matching according to the nodes which are able to provide resources in the network, when the nodes do not match the resources, the channel holding will be interrupted; in addition, although the algorithms of literature [25] and [27] conduct the bubbling matching for nodes density distribution, making the resource planning only in the way of resources matching by the best nodes is quite possible to result in serious congestion; consequently, the positioning time-delay index of the algorithm in this paper is lower than that of the control group.

\subsection{Influence of topology}

Different topologies have different influences on positioning error [28]; in this paper, the network topology described in literature [28] is taken as the test standard, where the network topology with general rules is the topological structure 1, the network topology of type C is the topological structure 2 , and the actually simulated

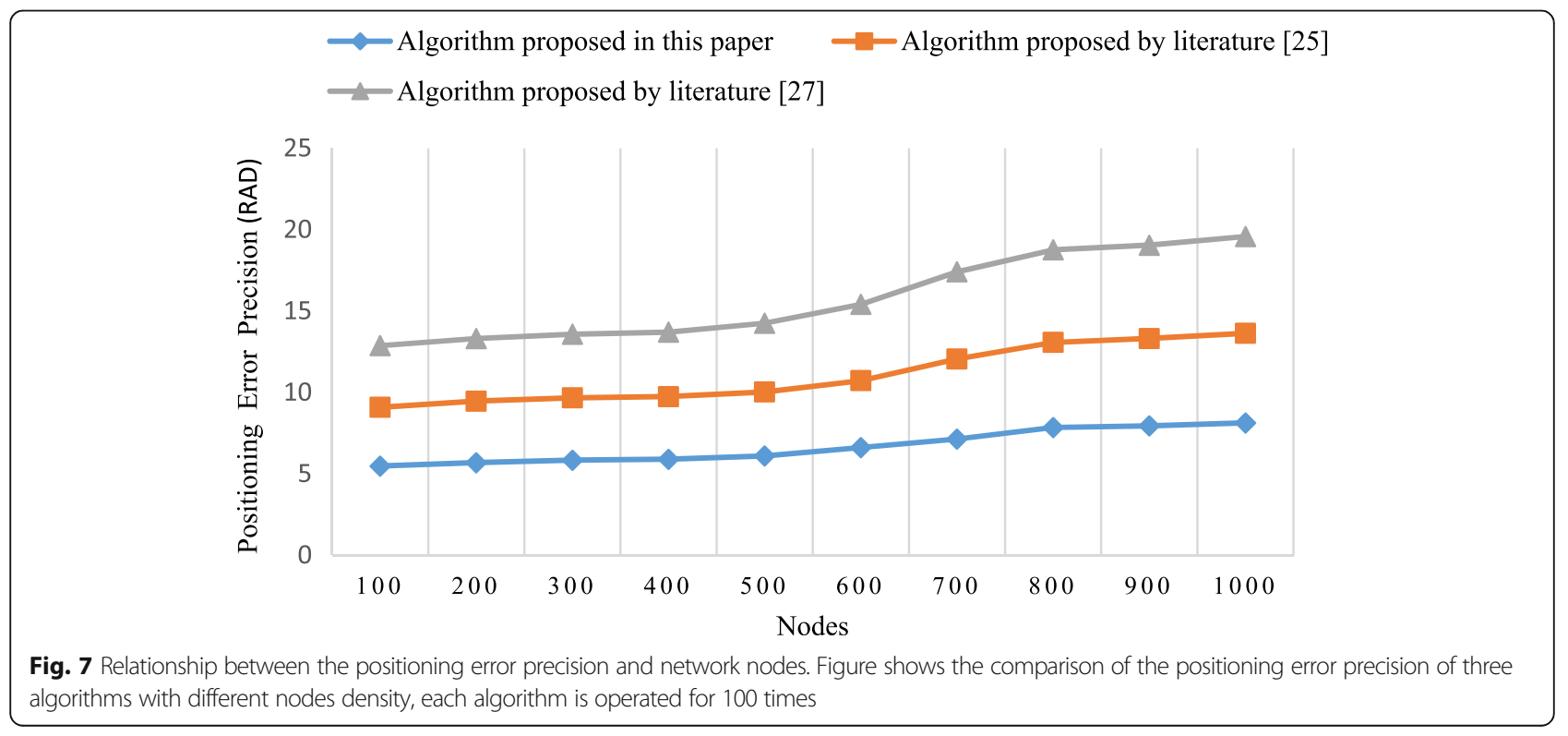




\section{-Algorithm proposed in this paper}

-Algorithm proposed by literature [25]

$\leftarrow$ Algorithm proposed by literature [27]

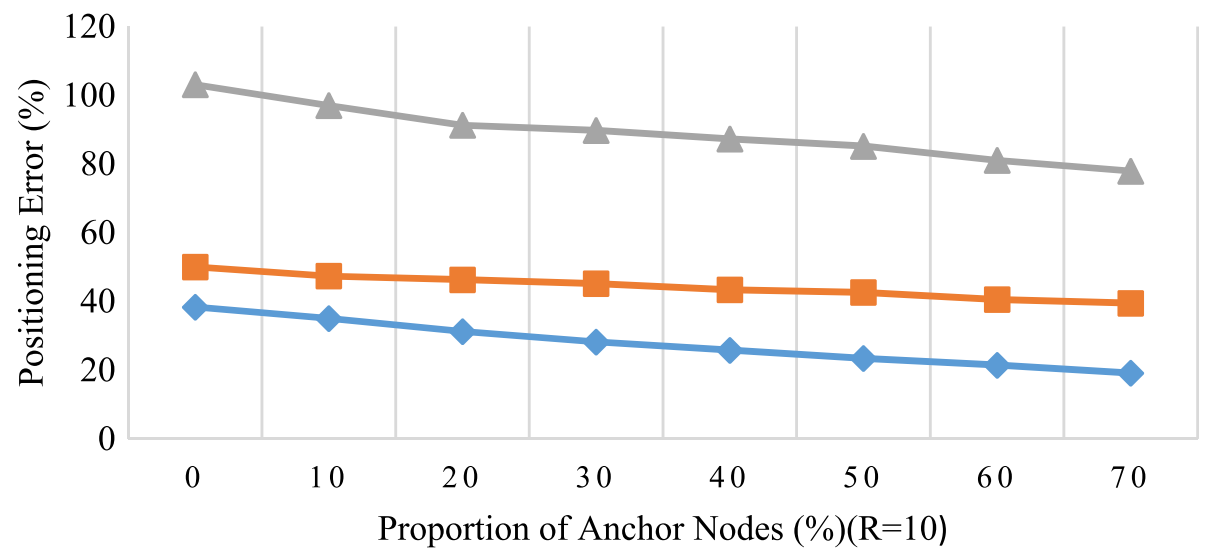

Fig. 8 Error change when proportion of anchor node at communication radius $r=10 \mathrm{~m}$. Figure shows the error change of three algorithms when proportion of anchor node at communication radius $r=10 \mathrm{~m}$, each algorithm is operated for 100 times

network topology is the topological structure 3. The algorithm in this paper, DV-HOP algorithm [29], and the algorithm in literature [27] are compared in the three topological structures, the results are shown in the following figures:

It can be found from Figs. 11, 12, and 13 that for the error rates under the three topological structures, the algorithm in this paper is advantageous and the positioning precision has been improved. In Fig. 11, the topological structure is good in performance, and the algorithm in this paper obviously helps improve the successful implementation. In Fig. 12, the nodes in topological structure are not distributed evenly, but the algorithm in this paper makes the average hop distance of each unknown node more conforming with the actual situation, and improved the success rate of node positioning. Figure 13 verified the positioning error in case of nodes being close to side boundary. It can be

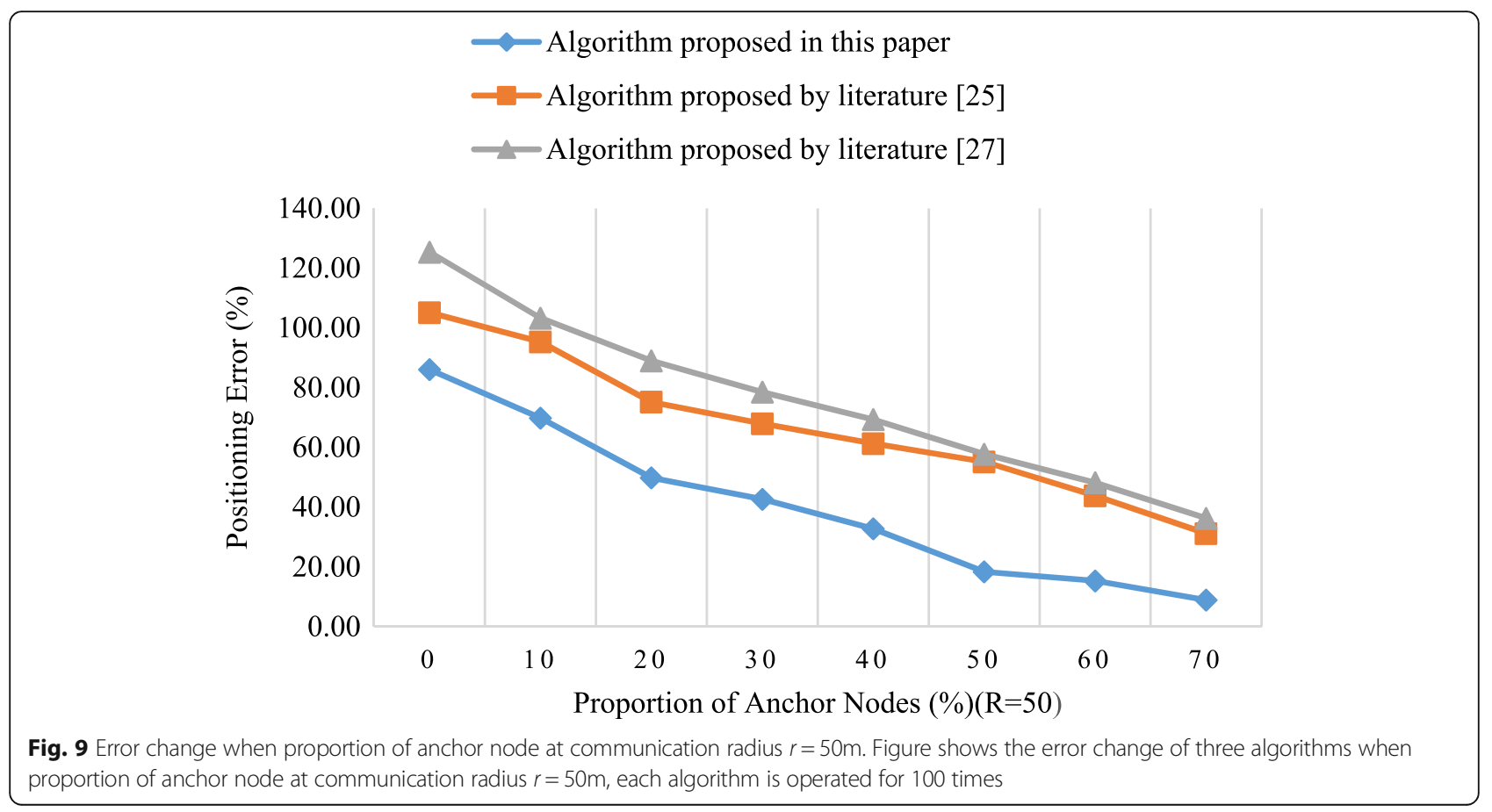



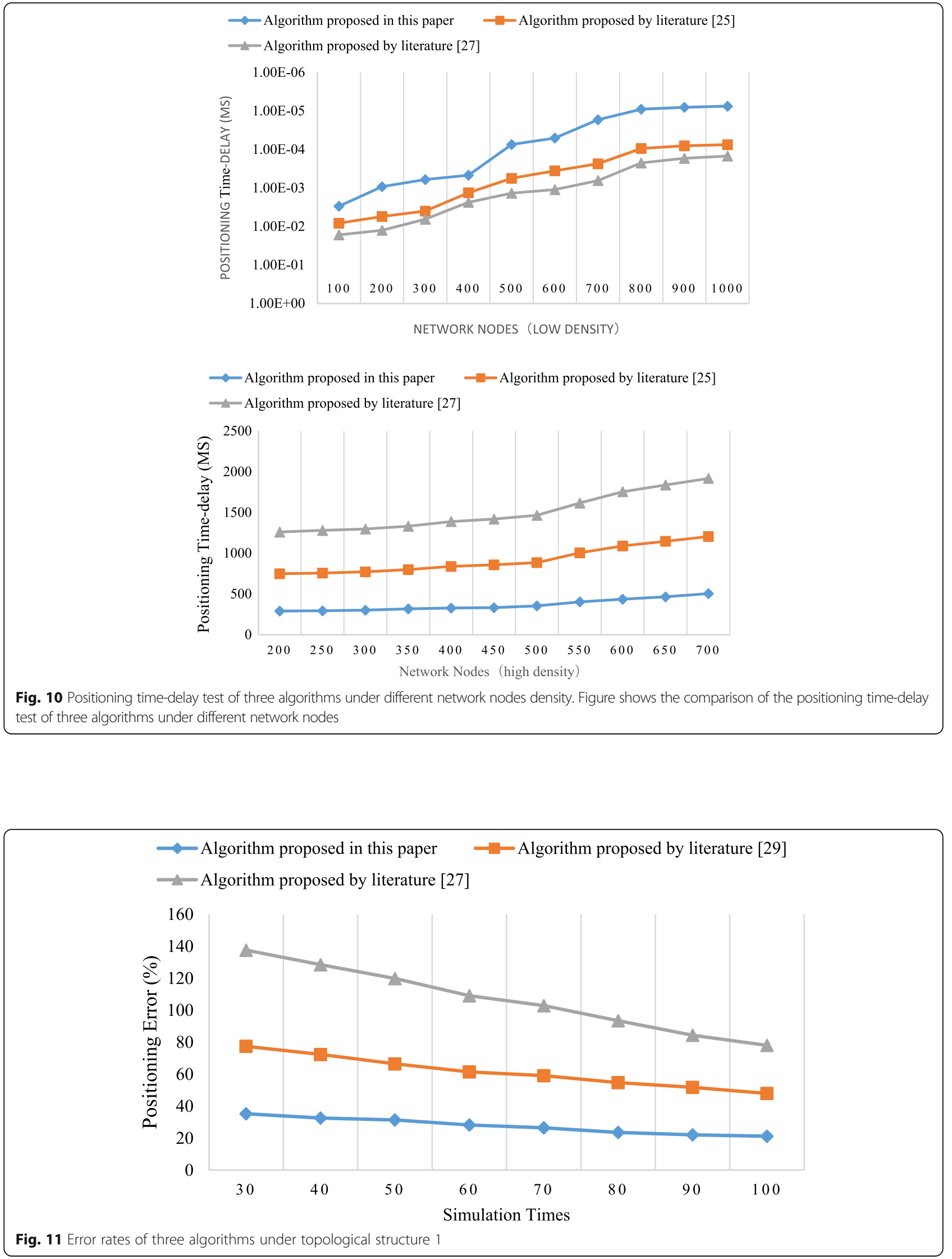


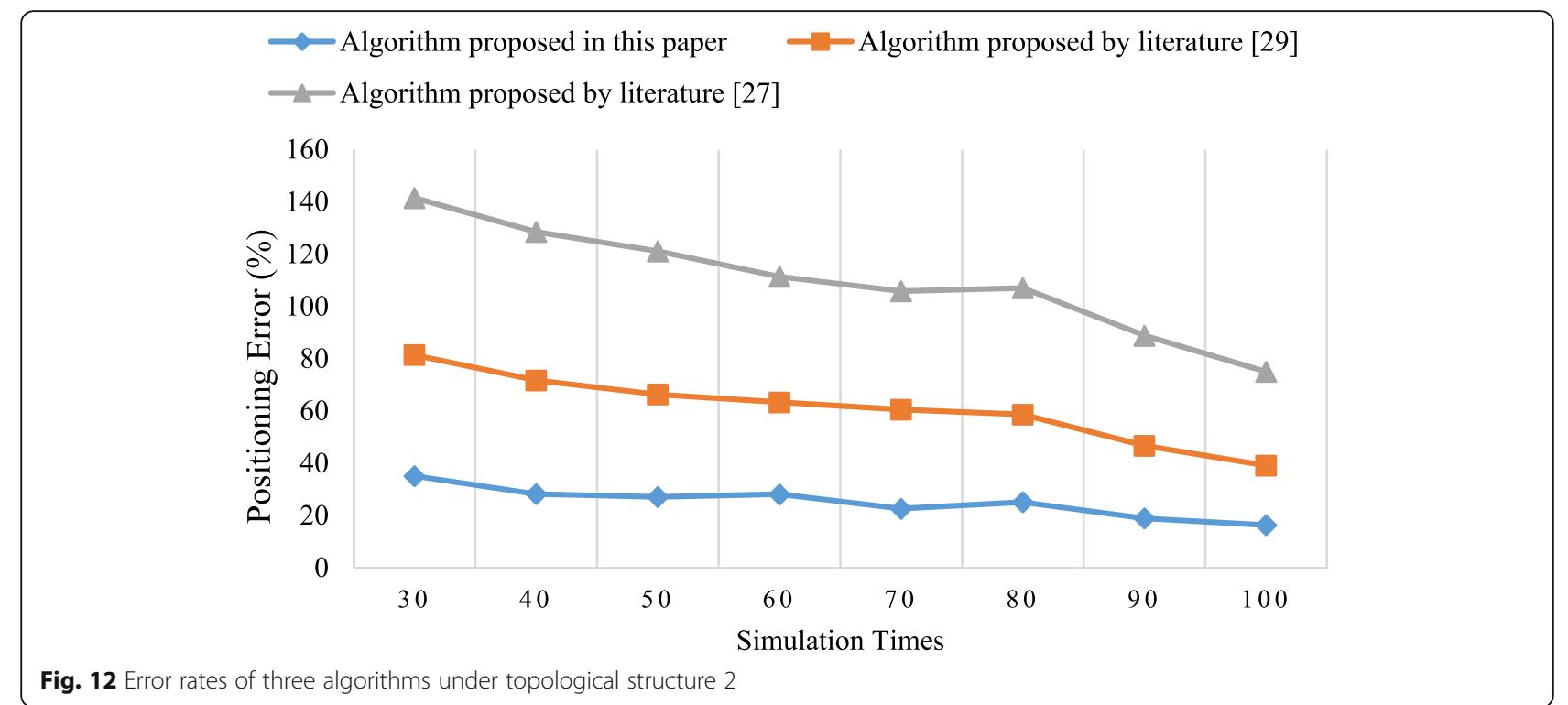

found that the algorithm in this paper resolved this problem effectively to a certain degree.

\subsection{Comparison of convergence performance}

Assuming that the distance measurement error is $20 \%$, the algorithm in this paper, particle swarm optimization (PSO) algorithm [26], and discrete PSO (DPSO) algorithm [31] are compared for convergence performance, and the simulation results are shown in Fig. 14. It can be found from Fig. 14 that with the increases of iteration times, at the initial moment, the fitness values of three algorithms have a sharp fall and tend to be stable after 10 iterations, which indicated that all the three algorithms are converged. The algorithm in this paper begins to converge after 12 iterations and shows better convergence performance, while the PSO algorithm begins to converge after 18 iterations and the DPSO algorithm begins to converge after 16 iterations, which proved that the algorithm in this paper is better than the PSO algorithm.

\subsection{Relationship between different iteration times and positioning error}

The algorithm in this paper is compared with the robust quadrilateral-based modified PSO (RQ-PSO) algorithm [30] and DPSO algorithm [31] in the experiment;

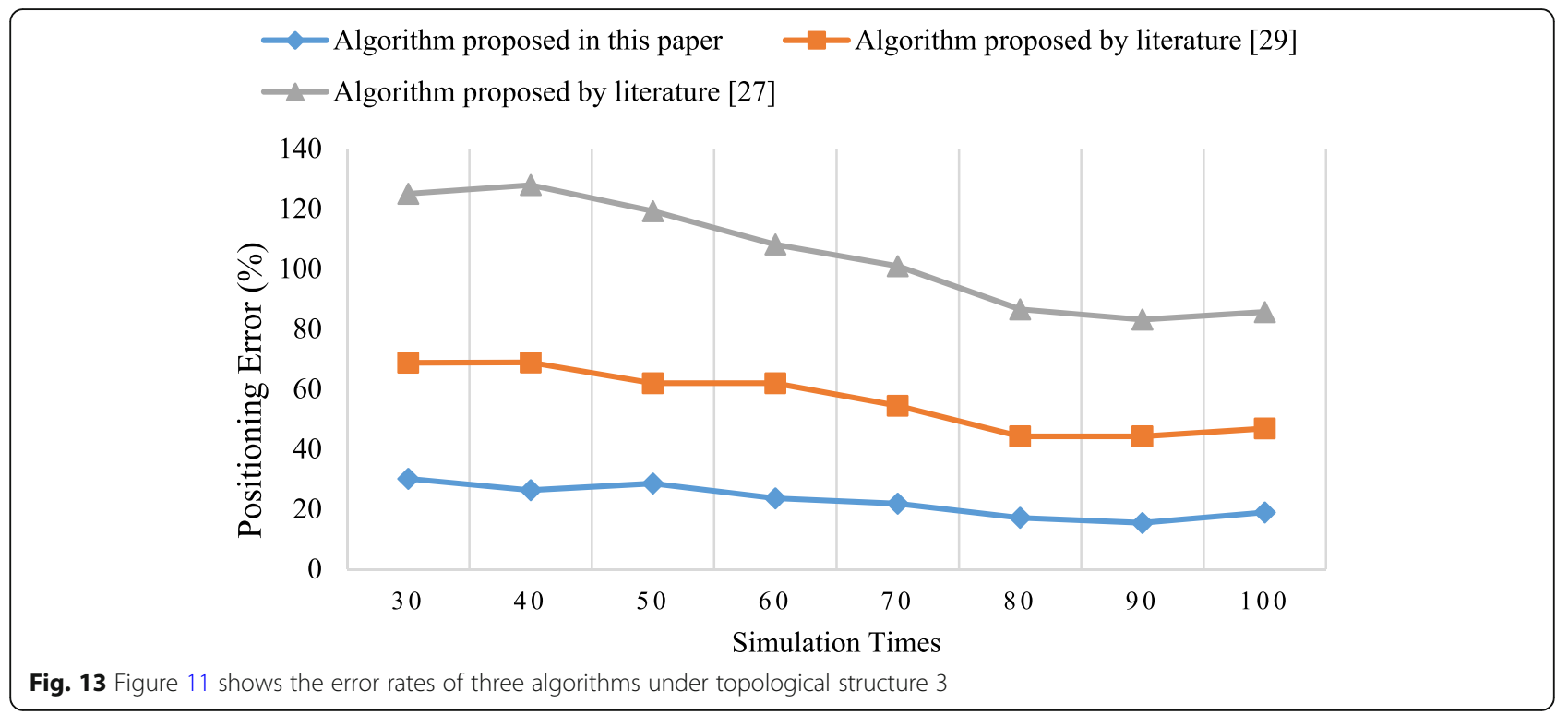




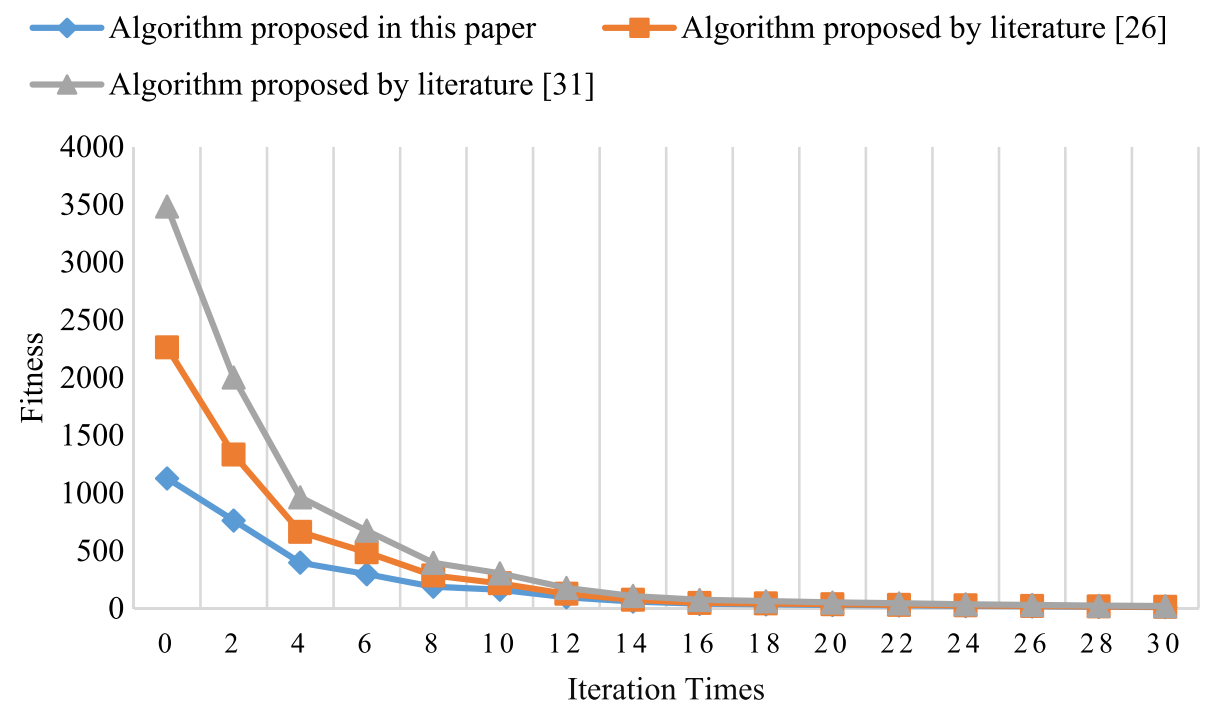

Fig. 14 Comparison of algorithms convergence performance

the relationship of their iteration times and the positioning error is shown in Fig. 15. It can be seen from the simulated picture that the positioning error of three algorithms reduces with the increases of iteration times; among them, the slope of curve of the algorithm in this paper is the biggest, that of the RQ-PSO is smaller, and that of the DPSO is the smallest. The algorithm in this paper tends to be stable after 14 iterations and the positioning error keeps being unchangeable, while the RQ-PSO algorithm keeps being stable after 18 iterations and the DPSO algorithm's positioning error keeps being unchangeable after 20 iterations. This proved that the convergence speed of the algorithm in this paper is faster than that of RQ-PSO algorithm and DPSO algorithm and has a proper positioning error.

\section{Conclusion}

The node positioning in wireless sensor network is always the focus of research. In this paper, on the basis of DV-HOP algorithm, the improvement was performed from aspects of improving the error precision and least square estimation. The simulation experiment demonstrated that the effect of the algorithm proposed in this paper is obvious, and in case of continuously increasing spending in hardware, the node positioning precision in wireless sensor network had been effectively improved;

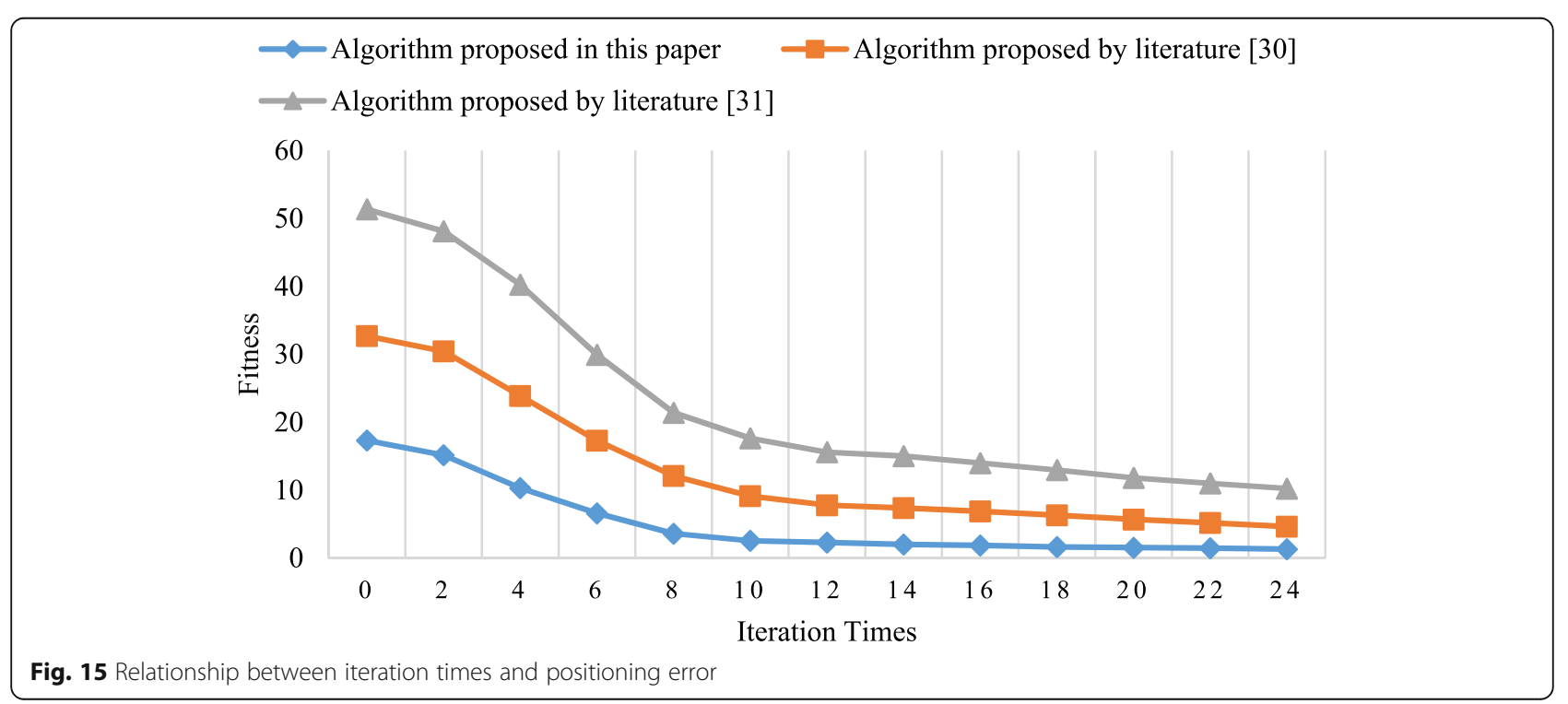


therefore, the estimated distance between unknown nodes and anchor nodes became more precise. In networks where little anchor nodes are necessary, the precise positioning for plenty of unknown nodes is realizable. The algorithm proposed in this paper has also a reference value for other positioning problems such as mobile communication.

\section{Abbreviations}

AOA: Angle of advent-based positioning algorithm; CPP: Colliner precision positioning algorithm; DPSO: Discrete PSO algorithm; DV-HOP: Distance VectorHOP; PSO: Particle swarm optimization; RQ-PSO: Robust quadrilateral-based modified PSO algorithm; RSSI: Received signal strength indicator-based algorithm: TDOA: Time difference of advent-based positioning algorithm; TOA: Time of advent-based positioning algorithm; WSN: Wireless sensor networks

\section{Acknowledgements}

Not applicable.

\section{Funding}

This project is supported by the Teaching Reform Project Fund of Scientific Research Fund of Guangdong Provincial Education Department under Grant (No: 416YCQ01); Teaching Reform Project Fund of Hunan province under Grant (No:2016[400]), (No:XJK17CXX002); Scientific Research Fund of Hunan Provincial Education Department under Grant (No:16C0299, 17C0295); the Natural Science Foundation of Hunan Province, China(Grant No.2018JJ2023); and Guangdong Science and Technology Plan Project under Grant 2016A020220003.

\section{Availability of data and materials}

Not applicable.

\section{Authors' contributions}

$T L$ is in charge of the major algorithm, and numerical simulations; the others were in charge of part of the theoretical analysis and experiment. All authors read and approved the final manuscript.

\section{Competing interests}

The authors declare that they have no competing interests.

\section{Publisher's Note}

Springer Nature remains neutral with regard to jurisdictional claims in published maps and institutional affiliations.

\section{Author details}

${ }^{1}$ College of Mechanical Electrical Engineering, University of Electronic Science and Technology of China, Zhongshan Institute, Zhongshan, China. ${ }^{2}$ School of Information and Electronic Engineering, Hunan City University, Yiyang, China.

\section{Received: 20 December 2018 Accepted: 25 April 2019}

\section{Published online: 22 May 2019}

\section{References}

1. D. Gong, Y. Yang, Z. Pan, Energy-efficient clustering in lossy wireless sensor networks. J. Parallel Distrib. Comput. 73(9), 1323-1336 (2013)

2. H. Dai, Q. Ye, G. Yang, et al., CSRQ: communication-efficient secure range queries in two-tiered sensor networks. Sensors 16(2), 1-17 (2016)

3. X. Zhang, L. Dong, H. Peng, et al., Collusion-aware privacy-preserving range query in tiered wireless sensor networks. Sensors 14(12), 23905-23932 (2014)

4. Y. Yao, N. Xiong, J.H. Park, L. Ma, J. Liu, Privacy-preserving max/min query in twotiered wireless sensor networks. Comput. Math. Appl 65(9), 1318-1325 (2012)

5. K. Wu, Y. Gao, F. Li, Y. Xiao, Lightweight deployment-aware scheduling for wireless sensor networks. Mobile Netw. 10(6), 837-852 (2005)

6. E. Aguirre et al., Design and implementation of context aware applications with wireless sensor network support in urban train transportation environments. IEEE Sensors J. 17(1), 169-178 (2017)

7. Y. Yoon, Y.H. Kim, An efficient genetic algorithm for maximum coverage deployment inwireless sensor networks. IEEE Trans. Cybern. 43(5), 14731483 (2013)
8. F.M. Al-Turjman, H.S. Hassanein, M.A. Ibnkahla, Efficient deployment of wireless sensor networks targeting environment monitoring applications. Comput. Commun. 36(2), 135-148 (2013)

9. J.-H. Chang et al., An efficient relay sensor placing algorithm for connectivity in wireless sensor networks. J. Inf. Sci. Eng. 27(1), 381-392 (2011)

10. L. Junfeng, P. Xiaogang, T. Lirui, Z. Yongchang, Using positioning priorities for accurate anchor-based node location over wireless sensor networks, Proceedings - 2017 IEEE 23rd international conference on parallel and distributed systems, ICPADS (2017), pp. 791-795

11. Z. Baoli, Y. Fengqi, in Proc. of IEEE International Conference on Informationand Automation. An energy efficient localization algorithmfor wireless sensor networks using a mobile anchor node (IEEE Press, Zhangjiajie, 2008)

12. S. Quanyi, Z. Jinfang, Z. Jie, Wireless sensor network node localization algorithm based on hopcount-angle factor. Comput. Eng. Des 38(4), 909915 (2017) (in Chinese)

13. Z. Ziwei, Z. Chao, A WS/N node localization algorithm combining centroid and DV-HOP algorithms. Comput. Appl. Soft. 32(11), 130-133 (2015)

14. N.B. Priyantha, H. Balakrishnan, E.D. Demaine, et al., Mobile Assisted Localization in Wireless Sensor Networks[C]//Proc. OfIEEE INFOCOM'05 (IEEE Press, Miami, 2005)

15. Z. Yan, Study of wireless sensor network localization algorithm based on RSSI. Comput. Sci. 36(4), 119-120 (2009)

16. B. Peng, L. Li, An improved localization algorithm based on genetic algorithm in wireless sensor networks. Cogn. Neurodyn. 9(2), 249-256 (2015)

17. Z. Fengmei, Z. Li, Research on improved passive target location algorithm in WSN. Appl. Res. Comput. 33(4), 1212-1215 (2016) in Chinese

18. J. Changjiang, T. Xianlun, X. Min, Unequal clustering routing protocol for wireless sensor networks based on PSO algorithm. Appl. Res. Comput. 29(8), 3074-3078 (2012) in Chinese

19. G. Zhongwen, G. Ying, H. Feng, et al., Perpendicular intersection: locating wireless sensors with mobile beacon. IEEE Trans. Veh. Technol. 59(7), 35013509 (2010)

20. F. Ruijuan, W. Qian, L. Qiang, Improved DV-HOP algorithm to WSN node localization on transmission line. Comput. Simul. 30(9), 131-134 (2013) in Chinese

21. L. Tu, Y. Zhang, X. Huang, L. Jia, A novel biological image encryption algorithm based on two-dimensional Feigenbaum chaotic map. J. Chem. Pharm Res 6(7), 2073-2082 (2014)

22. M. Yongyi, W. Yao, Encryption algorithm based on double chaos system with mutual feedback. J. Comput. Appl. 32(10), 2768-2770,2775 (2012) (in Chinese)

23. T. Li, Z. Chi, Z. Yingzheng, J. Liyuan, A novel image encryption algorithm based on two-dimensional general logistic mapping and output-feedback. J. Cent. South Univ. (Science and Technology) 45(6), 1893-1899 (2014) in Chinese

24. T.J.O. Kejian, Controlling the period-doubling bifurcation of logistic model. ActaPhysica Sinica 55(9), 4337-4441 (2006) in Chinese

25. B. Tang, X.R. Dong, High sensitivity GPS software receiver algorithm based on simple differential coherent accumulation. Signal Process. 25(5), 832-836 (2014)

26. Y. Peiru, X.S. Liang, Study on WSN node localization technology for environment monitoring. Comput. Sci.. 45(3), 94-99,125 (2018)

27. R. Shahryar, Oppsiton-based differential evolution. Evol. Comput. 12(1), 64 79 (2008)

28. G. Yang, J. Nightingale, Special Issue on Mobile Multimedia Big Data Embedded Systems, J. Adv. Comput. Intell. Intell. Inform. 22(7), 1071(2018)

29. Y. Lei, Y. Chengbo, Z. Songjian, T. Jun, L. Ru, WSN localization algorithm based on hop distance correction weighted DV-HOP. Piezoeledtrics\& Acoustooptics. 35(6), 899-906 (2013) in Chinese

30. C. Lujie, L. Mingyong, Z. Lichuan, S. Yongzhao, H. Shuai, A localization method for underwater wireless sensor network based on modified particle swarm optimization algorithms. J. Northwest. Polytech. Univ. 35(4), 648-654 (2017) in Chinese

31. M. Ling, L. Haijun, W. Chenggang, L. Guofeng, Optimal sensor placement based on improved discrete PSO algorithm. Acta Electron. Sin. 43(12), 24082413 (2015) in Chinese 